\title{
MICROBIOLOGICAL SPECTRE OF TERTIARY PERITONITIS AS A COMPONENT OF ITS DIAGNOSTICS AND TREATMENT
}

\author{
Petro Fomin \\ Department of surgery No. 3 \\ Bohomolets National Medical University \\ 13 T. Shevchenko blvd., Kyiv, Ukraine, 01601 \\ Oleh Matviychuk \\ Department of General surgery \\ Danylo Halytsky national medical university \\ 69 Pekarska str., Lviv, Ukraine, 79010 \\ oleh.matviychuk@yahoo.com \\ Olena Korniychuk \\ Department of Microbiology \\ Danylo Halytsky national medical university \\ 69 Pekarska str., Lviv, Ukraine, 79010
}

\begin{abstract}
The aim of the research was to investigate the microbial spectre of tertiary peritonits (TP) and its antibiotic resistance as the way to improve the diagnostics and treatment of TP.

Materials and methods. Prospective research enrolled 109 patients with secondary peritonitis. Tertiary peritonitis developed in $18,3 \%$ of cases. Samples of peritoneal exudate had been drawn upon index operation, relaparotomy and on the day of diagnosis of TP. Blood sampling was performed in patients with persistent fever, impaired consciousness, prolonged ( $\geq 4$ days) discharge from drainage tubes and on the 1st day of diagnosis of TP. Antibacterial susceptibility was evaluated using Hinton-Müller media.

Results and discussion. Patients were divided into 2 groups: with secondary peritonitis (89) and with TP (20). In TP group, cultivation of $76,2 \%$ of primary specimens resulted in replantable and identifiable growth, presenting a shift towards Gram-negative flora and higher incidence of Candida albicans. Following the development of TP, hemocultures were positive in $15,1 \%$, presented mainly by Proteus spp. and non-albicans Candida spp. Second-group carbapenems, tigecycline and piperacillin-tazobactam had shown the highest activity in pathogens of TP. Caspofungin proved to be the most potent antifungal agent, especially towards non-albicans Candida spp. Antibiotic resistance in TP group was marked in 63,8 \%.

Conclusions. Tertiary peritonitis is one of the most severe forms of abdominal sepsis with highest mortality. Causing pathogenic flora in case of TP is mainly Gram-negative and coccal with high rates of antibiotic resistance both in vitro and in vivo. Fungi, presented predominantly by Candida non-albicans substrains, show an increasing content in peritoneal exudate and major effect upon mortality in TP. In case of TP, a significant percent of peritoneal specimens do not provide any culture growth despite of observing stringent sampling, transportation and cultivation rules. Antimicrobial therapy of TP can never be standardized and should always be thoroughly based upon regular and proper peritoneal and blood sampling.
\end{abstract}

Keywords: abdominal sepsis, tertiary peritonitis, microbial spectre, fungi, antibacterial resistance.

\section{Introduction}

Abdominal sepsis remains to be the leading problem of modern emergency surgery despite the global progress of surgical and pharmaceutical technologies $[1,2]$. Tertiary peritonitis (TP) is one of the most severe forms of abdominal sepsis with dismal results of treatment, namely difficult verification of causal factors, mostly ineffective antibacterial treatment and, as a result, high rates of mortality [3, 4]. Alongside substantially impaired homeostasis, a major impact on severity of septic patient's condition is made by nosocomial microflora [5]. For decades, the latter presents high and fast increasing resistance to a vast array of antimicrobial agents, including potent and recently invented ones $[6,7]$. Clinicians frequently face the failure to cultivate and even to identify the microflora, nevertheless proper techniques and media are used [8,9]. Current studies show a di- 
versity of microbial spectre both of secondary peritonitis (SP) and TP, depending on region, type of department, nosology, antibiotic treatment regimen etc. [10]. Recently, an increasing role in course and prognosis of treatment of peritonitis is given to fungal infection [11]. All abovementioned prove the topicality of the problem of TP, its causing flora, antibiotic susceptibility and the need for their further in-depth investigation.

\section{Aim of the research}

The aim of the research was to investigate the microbial spectre of tertiary peritonits (TP) and its antibiotic resistance as the way to improve the diagnostics and treatment of TP.

\section{Materials and methods}

We had prospectively examined 109 patients with SP, operated in the Clinic of surgery and endoscopy of Lviv Danylo Halytsky national medical university in 2010-2015.

The etiology of SP was presented by: acute destructive appendicitis $(29,4 \%)$, perforated gastric $(11 \%)$ or duodenal $(7,3 \%)$ ulcer, perforated colon cancer $(11 \%)$, perforation of colonic diverticulitis $(6,5 \%)$, perforation of abdominal abscess $(5,6 \%)$, acute destructive cholecystitis with perforation $(4,7 \%)$, thrombosis of superior mesenteric artery with necrosis of the small intestine $(3,6 \%)$, perforated gastric cancer $(3,6 \%)$, Crohn's disease of the small intestine with perforation $(3,6 \%)$, hernia incarceration with necrosis of the small intestine $(2,8 \%)$, severe polytrauma with injury of the small bowel $(2,8 \%)$, decay of retroperitoneal tumor with formation of abscess and its perforation to abdomen $(1,8 \%)$, ingrowth of uterine tumor into small intestine and subsequent necrosis $(0,9 \%)$, pancreatic necrosis $(0,9 \%)$, volvulus of sigmoid colon with perforation $(0,9 \%)$, ulcerative colitis with necrosis of the colon $(0,9 \%)$, perforated cancer of the small bowel $(0,9 \%)$, decay of pancreatic cancer $(0,9 \%)$, gastric perforation by foreign body $(0,9 \%)$.

Concomitant somatic diseases, potentially aggravating the severity of condition, were detected in $88(80,7 \%)$ patients. Women slightly prevailed $-57(52,3 \%)$. The age of patients was within the range of $18-88$ (median - 61). Postoperatively, majority $(62,4 \%)$ of patients stayed for $1-4$ days (median - 2) in intensive care unit (ICU). Peritonitis was local non-separated in $104(95,4 \%)$, diffuse - in $88(80,7 \%)$ patients. Postoperative complications arose in 18 cases $(16,5 \%)$, being septic in $50 \%$.

Tertiary peritonitis was diagnosed on 3rd-12th day (median - 5) in 20 (18,3\%) patients. Criteria for diagnosis of TP were: persistence of peritoneal symptoms despite adequate surgical elimination of the infectious source, presence of nosocomial microflora in peritoneal exudate, multi-organ failure and time of stay in intensive care unit for $\geq 3$ days [12]. Postoperative mortality in the whole cohort was 30,2\%. Tertiary peritonitis had a lethal outcome in $90 \%$ of cases, with sepsis being the main cause.

Samples of peritoneal exudate had been drawn upon index operation for SP at least from 4 distant areas in case of diffuse peritonitis and from 2 in case of local each using a separate swab and container with protective environment. All specimens had been transported to microbiological laboratory within $15 \mathrm{~min}$ and were cultivated using different nutritional media in appropriate thermal environment. In case of programmed relaparotomy (PRL) / on-demand relaparotomy (ODRL) and on the day of diagnosis of TP, we had additionally performed sampling of peritoneal exudate directly from the peritoneal cavity and/or drainage tubes at least from 2 remote locations.

Blood sampling was performed from both cubital veins in all patients with persistent $(\geq 48 \mathrm{~h})$ fever, impaired consciousness (according to Glasgow scale values), prolonged ( $\geq 4$ days) discharge from drainage tubes and on the 1st day of TP diagnosis. In case blood samples gave growth to skin saprophytes, the sampling was repeated once again from both cubital veins.

Actively growing colonies were identified by microscopy and/or enzyme technique. Gram-positivity and primary phenotyping of flora were evaluated by cultural features and biochemical identification systems after $24 \mathrm{~h}$. Identified cultures had been replanted onto Hinton-Müller media to evaluate susceptibility to 19 antibiotics using Kirby-Bauer method. Susceptibility of pathogenic fungi to fluco- and voriconasole was evaluated on glucose-enriched agar using semi-quantitative method. Candida and its subspecies were identified using mannan and galactomannan serological method. 
In majority of cases $(98,4 \%)$, initial antibacterial therapy consisted of 2 drugs with $1^{\text {st }}$ either fluoroquinolone $\left(3^{\text {rd }}\right.$ generation $\left.-53 \%, 4^{\text {th }}-47 \%\right)$, cephalosporin $\left(3^{\text {rd }}\right.$ generation $-46,3 \%, 4^{\text {th }}-$ $53,7 \%)$ or ertapenem $(10,4 \%) ; 2^{\text {nd }}$ - anti-anaerobic agent $(44,2 \%$ - metronidazole, $55,8 \%$ - ornidazole). Antifungal drugs were prescribed pre-emptively before initial operation to $12,2 \%$ patients (fluconazole $-68,1 \%$, voriconazole $-31,9 \%$ ). None of the patients had clinical signs of fungal infection upon admission to the hospital.

\section{Results}

Patients were divided into 2 groups: with SP ( $n=89)$ and with TP $(n=20)$. In SP group, cultivation of $85,7 \%$ of primary specimens resulted in replantable and identifiable growth, in TP group $-76,2 \%$. As seen in Table 1, aerobic flora had quantitatively prevailed in primary specimens of SP. In those patients, who developed TP further on, a shift towards Gram-negative flora was marked alongside higher incidence of Candida albicans.

Table 1

Microbial spectre of SP and TP upon index operation

\begin{tabular}{|c|c|c|}
\hline Strain & SP, $\%$ & TP, \% \\
\hline \multicolumn{3}{|l|}{ Gram-positive } \\
\hline Staphylococcus epidermidis & 45,3 & 43,5 \\
\hline Methicillin-sensitive Staphylococcus aureus & 15,6 & 12,1 \\
\hline Methicillin-resistant Staphylococcus aureus & 1,2 & 2,4 \\
\hline Enterococcus faecalis & 28,1 & 26,7 \\
\hline Streptococcus pyogenes & 32,3 & 36,5 \\
\hline Streptococcus viridans & 15,6 & 17,2 \\
\hline \multicolumn{3}{|c|}{ Gram-negative } \\
\hline Escherichia coli & 37,8 & 44,3 \\
\hline Pseudomonas aeruginosa & 7,9 & 13,2 \\
\hline Citrobacter Freundii & 31 & 28,7 \\
\hline Klebsiella mobilis & 42,2 & 37,3 \\
\hline Klebsiella pneumoniae & 4,1 & 5,5 \\
\hline Proteus vulgaris & 11,9 & 10,1 \\
\hline Acinetobacter spp. & 66,4 & 49,8 \\
\hline Moraxella spp. & 12,5 & 9,7 \\
\hline Serratia spp. & 9,8 & 11,2 \\
\hline \multicolumn{3}{|c|}{ Fungi } \\
\hline Candida albicans & 1,7 & 7,6 \\
\hline Candida glabrata & 0 & 6,2 \\
\hline Candida krusei & 0 & 5,5 \\
\hline Candida tropicalis & 0 & 1,5 \\
\hline Geotrichum candidum & 1,1 & 4,7 \\
\hline
\end{tabular}

A detailed analysis of primary microbiograms had shown that the infection was presented by cultural associations. In SP group, 3 different microorganisms formed an association in 24,5\% of cases, 2 - in 70,1\% and only in 5,4\% it was monocultural. Microbial associations in the TP group were alike to those in SP group, but with a 23,1\% fraction of Candida spp.

Relaparotomy, regardless PRL or ODRL, was the indication for peritoneal exudate sampling. In case of PRL, volume of peritoneal fluid was scarce and so were the findings (positive growth $-51,4 \%)$ : a variety of coccal flora $(88,3 \%$ of cultures), associated with $E$. coli $(14,3 \%$ of cases). In contrast, specimens taken during ODRL presented a wider (89,5\%) range of microorganisms, incl. E. coli, Klebsiella spp., Proteus spp. and Enterobacter spp., associated with Candida albicans in $13,1 \%$ of cases.

In $58,7 \%$ of patients, TP was diagnosed on ODRL, in $15,4 \%$ - on PRL. Intraoperational sampling was positive only in $13,7 \%$, from drainages - in 11,2 \%. Cumulative microbial spectre of TP is shown in Table 2. 
Table 2

Microbial spectre of TP

\begin{tabular}{|c|c|}
\hline Strain & $\%$ \\
\hline \multicolumn{2}{|c|}{ Gram-positive } \\
\hline Staphylococcus epidermidis & 12,9 \\
\hline Staphylococcus saprophyticus & 10 \\
\hline Methicillin-sensitive Staphylococcus aureus & 7,4 \\
\hline Methicillin-resistant Staphylococcus aureus & 34,4 \\
\hline Enterococcus faecalis & 20,1 \\
\hline Enterococcus faecium & 11,1 \\
\hline Streptococcus pyogenes & 26,1 \\
\hline Streptococcus viridans & 13,6 \\
\hline \multicolumn{2}{|c|}{ Gram-negative } \\
\hline Escherichia coli & 25,9 \\
\hline Pseudomonas aeruginosa & 44,6 \\
\hline Citrobacter Freundii & 28,7 \\
\hline Klebsiella pneumoniae & 27,1 \\
\hline Klebsiella oxytoca & 12,2 \\
\hline Klebsiella mobilis & 43,5 \\
\hline Proteus vulgaris & 24 \\
\hline Proteus mirabilis & 11,2 \\
\hline Acinetobacter Baumannii & 33,5 \\
\hline Moraxella spp. & 15,1 \\
\hline Serratia spp. & 9,4 \\
\hline \multicolumn{2}{|c|}{ Fungi } \\
\hline Candida albicans & 15,3 \\
\hline Candida glabrata & 23,1 \\
\hline Candida krusei & 26,4 \\
\hline Candida tropicalis & 14,6 \\
\hline Geotrichum candidum & 2,7 \\
\hline
\end{tabular}

Among patients of TP group, whose peritoneal exudate gave growth to Candida spp. ( $90 \%$ non-albicans), $80 \%$ died.

Initial blood sampling was successful in $41,2 \%$ of SP cases, though being false-positive in 9,3\%. Pathogenic flora was verified in 35,4\% cases and was presented by Streptococcus pyogenes (23,4 \%), E. coli (10,3\%), Proteus vulgaris (34,1 \%) and Acinetobacter Baumanii (32,2\%), not influencing the previously applied antibiotherapy. Following the development of TP, hemocultures were positive in 15,1\%, presented mainly by Proteus spp. and non-albicans Candida spp.

Taking into account a multifold amount of results, antibacterial susceptibility to chosen drugs in SP group is given as Expected clinical efficacy (ECE) ( $\geq 66 \%$ of cultures presented delayed growth $\geq 20 \mathrm{~mm}$ ) in Fig. 1 .

In SP group, caspofungin had shown the highest antifungal ECE (23\%). Voriconazole had ECE $22,8 \%$, fluconazole $-17,8 \%$.

Expected clinical efficacy in TP group is shown in Fig. 2.

We had observed a substantial shift of Candida towards non-albicans subspecies, which in $68,2 \%$ had exposed a good susceptibility to caspofungin $(26,1 \%)$ and voriconazole $(18,3 \%)$ and a rather poor one to fluconazole $(9,5 \%)$. Half of the TP group received 4 antimicrobials at once incl. 1 systemic antifungal agent.

Noteworthy peculiarities have also been marked upon analysis of ECE values depending on Gram-polarity of pathogens (Table 3). In most cases of TP, antibacterials showed lower ECE cf. to SP group. 


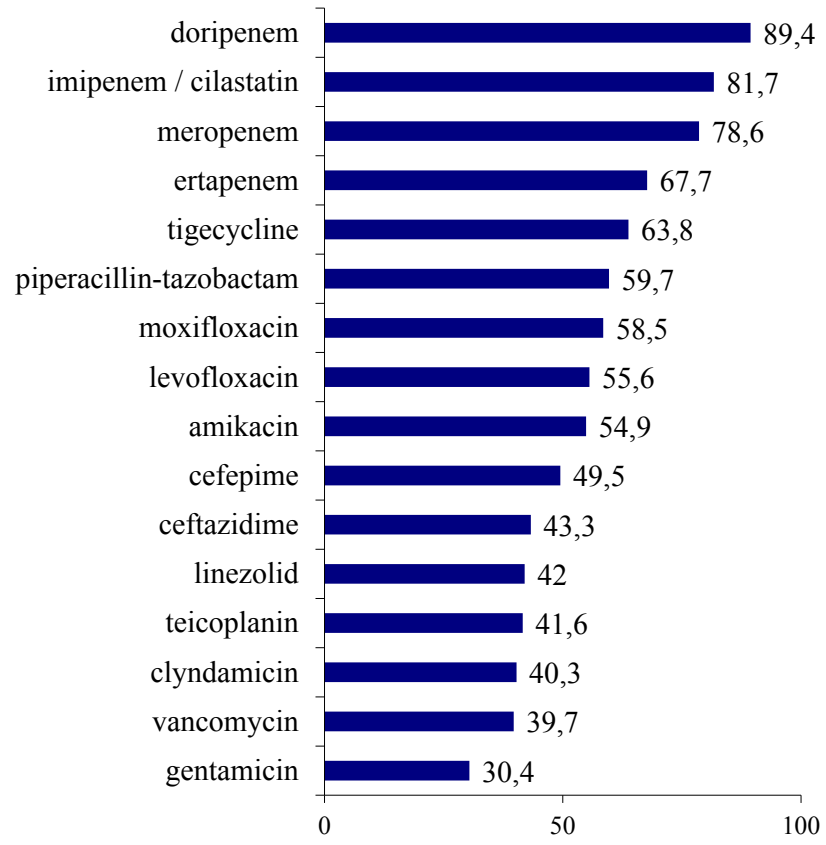

Fig. 1. ECE - SP group ( $\%$ of sensitive cultures)

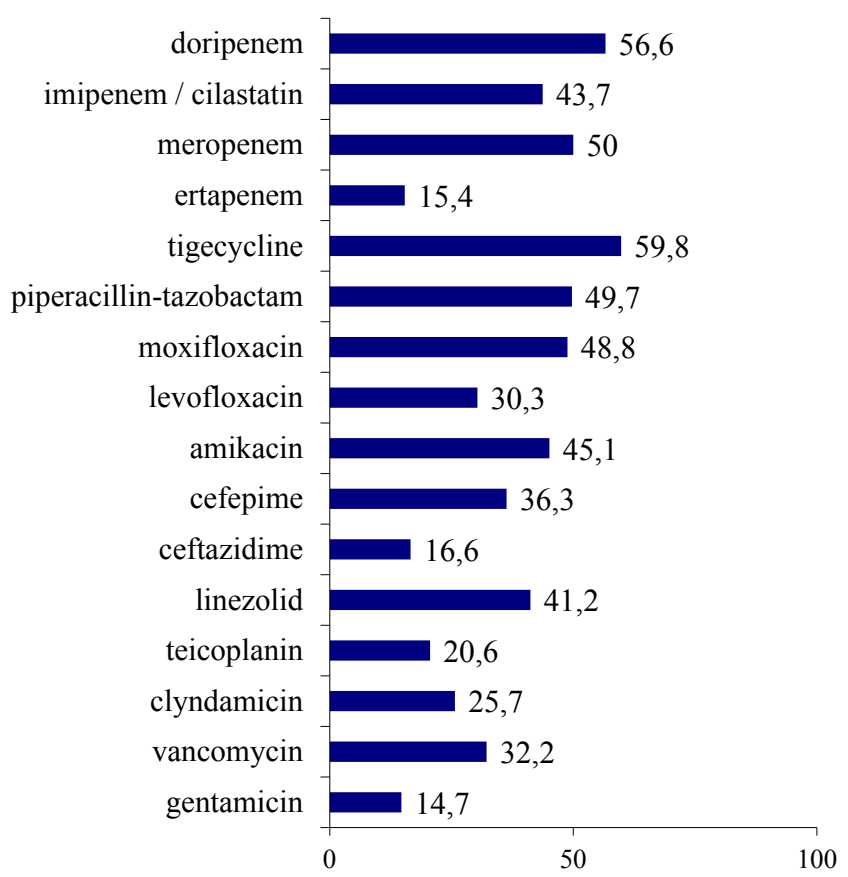

Fig. 2. ECE - TP group ( $\%$ of sensitive cultures)

Antibiotic resistance to $\geq 2$ antibiotics was revealed in $35,3 \%$ patients of SP group with prevalence of extended spectrum $\beta$-lactamase producing pathogens (Enterobacteriaceae, Pseudomonas aeruginosa). In TP group this parameter was 63,8 \%: $2^{\text {nd }}$ group carbapenem-resistant Acinetobacter baumanii (61,3\% of cultures), methicillin-resistant Staphylococcus aureus (32,1\%), fluconasole-resistant Candida albicans (58,3\%). In general, multi-drug resistant (MDR) pathogens comprised $28,7 \%$ of cases in TP group and were represented mainly by Acinetobacter spp. and Klebsiella spp. with highest (39,8\%) sensitivity to doripenem.

Received results created the basis for introduction of changes in antimicrobial treatment (Table 4). 
Table 3

ECE depending on Gram-polarity (\% of sensitive cultures)

\begin{tabular}{ccccc}
\hline Antibacterial drug & \multicolumn{2}{c}{ Gram-positive } & \multicolumn{2}{c}{ Gram-negative } \\
& SP & TP & SP & TP \\
\hline doripenem & 80 & 68 & 92 & 81 \\
imipenem / cilastatin & 76 & 65 & 84 & 76 \\
meronem & 78 & 63 & 80 & 69 \\
ertapenem & 60 & 41 & 70 & 37 \\
tigecycline & 64 & 58 & 69 & 58 \\
piperacillin-tazobactam & 75 & 62 & 30 & 21 \\
moxifloxacin & 86 & 74 & 48 & 39 \\
levofloxacin & 50 & 59 & 65 & 48 \\
amikacin & 57 & 38 & 62 & 54 \\
cefepime & 30 & 42 & 55 & 31 \\
ceftazidime & 68 & 50 & 34 & 15 \\
linezolid & 50 & 41 & 11 & 10 \\
teicoplanin & 51 & 32 & 9 & 8 \\
clindamycin & 50 & 28 & 13 & 5 \\
vancomycin & 45 & 43 & 45 &
\end{tabular}

Table 4

Characteristics of antimicrobial treatment in both groups

\begin{tabular}{|c|c|c|c|c|}
\hline Type of antibiotic treatment & \multicolumn{2}{|c|}{ SP } & \multicolumn{2}{|c|}{$\mathbf{T P}$} \\
\hline de-escalation & \multicolumn{2}{|c|}{$14,3 \%$} & \multicolumn{2}{|c|}{$0 \%$} \\
\hline escalation & \multicolumn{2}{|c|}{$4,7 \%$} & \multicolumn{2}{|c|}{$100 \%$} \\
\hline combined & $85,6 \%$ & $\begin{array}{c}93,3 \%-2 \text { agents } \\
6,7 \%-3 \text { agents }\end{array}$ & $100 \%$ & $\begin{array}{c}73,1 \%-2 \text { agents, } \\
16,6 \%-3 \text { agents, } \\
10,3 \%-4 \text { agents }\end{array}$ \\
\hline definitive & \multicolumn{2}{|c|}{$44,5 \%$} & \multicolumn{2}{|c|}{$30 \%$} \\
\hline
\end{tabular}

It is worth of mentioning that several substrains of Staphylococci (TP group) demonstrated medium (33-66 \%) susceptibility to fosfomycin. As well, we observed $\geq 66 \%$ in vitro efficacy of co-trimoxazole towards certain strains of Acinetobacter spp. and Citrobacter spp. Similar in vitro data were obtained about activity of colistin towards P. aeruginosa and E. coli. Although, none of those were chosen for use in vivo due to known significant side effects, particularly toxicity and risk of further promotion of antibiotic resistance.

\section{Discussion}

Tertiary peritonitis was once called an "uncontrolled peritonitis", manifesting itself as sepsis at the background of sterile peritoneum [13]. Hospital-acquired infections, concomitant diseases and immunosuppression are one of the most potent risk factors of mortality [14]. Failure of antibacterial therapy is considered to be one of the indications for hemoculturing, as Candida spp. are often suspected [9]. Severity of condition, causal pathogenic flora and prognosis differ substantially in septic patients of surgical department and ICU [15].

According to a recent study of O. van Ruler et al. [16], $70 \%$ of peritoneal cultures of SP were polymicrobial, $19 \%$ - monomicrobial and $11 \%$ showed no microbial growth. Another group of authors observed $72 \%$ cases of polymicrobial, $8-28 \%$ monomicrobial cultures and up to $20 \%$ of unsuccessful microbial sampling $[17,18]$. These data partially coincide with ours, though percentages in TP group differ essentially.

Recent studies of microbial spectre of SP prove that it is mostly stable throughout years: E. coli $-50-100 \%$, Streptococcus spp. - 10-44\%, P. aeruginosa $-24,9 \%$, S. aureus $-16 \%$ [19]. Severity of the patient's condition and difficulties of adequate "source control" upon index emergency operation often form the indication for relaparotomy - a known risk factor for further colonization of abdominal cavity by nosocomial pathogens [20]. This could explain why causal flo- 
ra of TP is alike to that in SP, though has obvious qualitative and quantitative differences. Majority of modern works indicate on lower incidence of successful peritoneal sampling, a move towards Gram-positive flora and increasing content of fungi [21]. One study observed E. coli in $52 \%$ cultivates of TP, Klebsiella spp. - $10 \%$, Enterobacter spp. - $19 \%$, P. aeruginosa $13 \%$, Enterococcus faecalis - $33 \%$, Enterococcus faecium - $8 \%$ [22]. These findings also have similarities with our research, though, study duration could have led to changes both in spectre and antibiotic susceptibility over time. Diversities and sometimes discrepancies in microbial spectres of peritonites could be explained by differences in regional protocols/standards of antibiotic treatment and structure of morbidity of given population.

Yeast strains comprise $22-41 \%$ in exudate of SP and $17 \%$ of all nosocomial isolates in ICU [23]. Isolation of Candida from intra-abdominal cultures is successful in $57 \%$ of cases and is associated with increased mortality [24]. In the structure of obtained fungal cultures during SP, Candida albicans was seen in $74 \%$, Candida glabrata - $17 \%$; remaining $9 \%$ included: Candida inconspicua, Candida parapsilosis, Candida tropicalis, Candida zeylanoides, Geotrichum candidum [23]. In our research, in SP group we observed lesser percentage of Candida albicans and very moderate - of Candida non-albicans in comparison to TP group.

Prevalence of Candida, especially of its non-albicans strains, in peritoneal exudate on index operation hints upon compromised anti-infective defense and latent immunodeficiency as potent risk factors for development of TP. In case of candidous TP, mortality reaches $70 \%$ [11]. Peritoneal sampling during TP had shown positive culturing of albicans-subspecies in $12 \%$ of cases, non-albicans-subspecies - in $3 \%$ [23]. In TP group, we had seen a remarkable shift of the ratio of "albicans/non-albicans" subspecies in favour of the latter.

Blood culture is a necessary method to control the spread of the infection to the systemic level. According to reports, it's successful in 9,2-21,2 \% [25, 26]. Results of hemocultivation differ as well, probably depending on patient's general health condition, severity of surgical disease, characteristics of therapy and accuracy of cultivation. Leading Gram-positive pathogens in blood culture are Staphylococcus spp. (48,7\%) and Enterococcus spp. (12,8\%) [10]. Gram-negative pathogens were obtained from $25,7 \%$ specimens and included Klebsiella pneumoniae (12,8 \%), Enterobacter cloacae (2,6 \%), E. coli (2,6\%), Proteus vulgaris (2,6\%); others (7,7\%) were: P. aeruginosa (2,6\%), Acinetobacter baumannii (2,6\%) and Candida (2,6\%) [10]. In our study, mostly Gram-positive coccal flora was found in blood of patients of SP group and only 2 pathogens in TP group, notably, non-albicans Candida spp.

International research data claim a vast range of hemocultivation of Candida spp. - 4-32\% [27]. Regardless of primary disease, Hung-Wei C. et al. [28] reported a shift from Candida albicans to Candida non-albicans subspecies in hemocultures, with non-albicans quotient equaling up to $63 \%$. Out results of hemocultivation in TP group were $90 \%$ of non-albicans substrains.

Clinicians still deal with unclear previous history of antibiotic use and fungal status, which directly affect the results of antibacterial treatment. Normally, antibacterial therapy should start as pre-emptive, later on transforming into a definitive one. Nowadays, there are probably no borders for migration of resistant microflora and interchange of its defense mechanisms between species. Even if "source" and "damage control" principles had been maintained on the index procedure, inadequate antimicrobial therapy is the risk factor for pessimistic outcome [21].

Antimicrobial susceptibility data vary a lot due to the huge set of independent factors, including technique of sampling, quality of nutritional media, subjectivity of interpretation and actual antimicrobial protocols. In our study, $2^{\text {nd }}$ group carbapenems showed the highest in vitro efficacy in both study groups, corresponding to data of other colleagues [29]. Other clinicians call to diminish/ignore the use of any generation cephalosporins due to serious drop of their efficacy with time and strong promotion of antibacterial resistance [26].

On one hand, antifungal therapy should be prescribed either to each critical patient or in case of positive fungal culture [8], on the other - empiric coverage of yeast in abdominal sepsis is not supported by present data because of high resistance of Candida spp. to fluconasole [27].

Finally, the "collateral damage" effect, alongside natural process of antibiotic resistance, became the main reason of emergence of MDR pathogens. Antibiotic resistance to $\geq 2$ antibiotics 
occurred in $64,9 \%$ of cases of SP, presented mainly by extended spectrum $\beta$-lactamase-producing Enterobacteriaceae spp. and P. aeruginosa in $11,1 \%$ and $11,9 \%$ respectively [26]. In other works, fraction of nosocomial MDR reached $84,8 \%$, but just $14 \%$ in case of TP [23]. Our findings of MDR pathogens make up for $35,3 \%$ in SP group, and 28,7 \% of strains in TP group.

All above emphasize the complexity of the problem of TP and the necessity of further indepth investigation of its microbial spectre and antibiotic resistance in order to improve the approach to diagnostics and treatment results.

\section{Conclusions}

1. Tertiary peritonitis remains to be one of the most severe forms of abdominal sepsis with highest mortality.

2. Causing pathogenic flora in case of tertiary peritonitis is mainly Gram-negative and coccal with high rates of antibiotic resistance both in vitro and in vivo.

3. Fungi, presented predominantly by Candida non-albicans substrains, show an increasing content in peritoneal exudate and major effect upon mortality in tertiary peritonitis.

4. In case of tertiary peritonitis, a significant percent of peritoneal specimens do not provide any culture growth despite of observing stringent sampling, transportation and cultivation rules.

5. Antimicrobial therapy of tertiary peritonitis can never be standardized and should always be thoroughly based upon regular and proper peritoneal and blood sampling.

\section{References}

[1] Kamble, R., Singh, M., Jaiswal, Y. (2016). Prognostic factors in perforative peritonitis: an observational study. International Surgery Journal, 3 (3), 1082-1092. doi: 10.18203/2349-2902.isj20162232

[2] Kon, K., Rai, M. (2014). Microbiology for Surgical Infections: Diagnosis, Prognosis and Treatment. Amsterdam: Elsevier, 324. doi: 10.1016/c2012-0-07539-4

[3] Ballus, J., Lopez-Delgado, J. C., Sabater-Riera, J., Perez-Fernandez, X. L., Betbese, A. J., Roncal, J. A. (2017). Factors Associated with the Development of Tertiary Peritonitis in Critically Ill Patients. Surgical Infections, 18 (5), 588-595. doi: 10.1089/sur.2016.018

[4] Chromik, A. M., Meiser, A., Holling, J., Sulberg, D., Daigeler, A., Meurer, K. et. al. (2009). Identification of Patients at Risk for Development of Tertiary Peritonitis on a Surgical Intensive Care Unit. Journal of Gastrointestinal Surgery, 13 (7), 1358-1367. doi: 10.1007/s11605-009-0882-y

[5] Panhofer, P., Izay, B., Riedl, M., Ferenc, V., Ploder, M., Jakesz, R., Gotzinger, P. (2009). Age, microbiology and prognostic scores help to differentiate between secondary and tertiary peritonitis. Langenbeck's Archives of Surgery, 394 (2), 265-271. doi: 10.1007/s00423-008-0301-y

[6] Diaz-Martin, A., Martinez-Gonzalez, M., Ferrer, R., Ortiz-Leyba, C., Piacentini, E. et. al. (2012). Antibiotic prescription patterns in the empiric therapy of severe sepsis: combination of antimicrobials with different mechanisms of action reduces mortality. Critical Care, 16 (6), R223. doi: 10.1186/cc11869

[7] Dellinger, R. P., Levy, M. M., Rhodes, A., Annane, D., Gerlach, H. et. al. (2013). Surviving Sepsis Campaign: International Guidelines for Management of Severe Sepsis and Septic Shock, 2012. Intensive Care Medicine, 39 (2), 165-228. doi: 10.1007/s00134-012-2769-8

[8] Chow, A. W., Evans, G. A., Nathens, A. B., Ball, C. G., Hansen, G., Harding, G. K. et. al. (2010). Canadian Practice Guidelines for Surgical Intra-Abdominal Infections. Canadian Journal of Infectious Diseases and Medical Microbiology, 21 (1), 11-37. doi: 10.1155/2010/580340

[9] Winter, V., Czeslick, E., Sablotzki, A. (2007). Sepsis and multiple organ dysfunctions: pathophysiology and the topical concepts of treatment. Anesteziol. Reanimatol., 5, 66-72.

[10] Beloborodova, N. V., Vostrikova, T. Yu., Chernevskaya, E. A. (2008). Etiology of postoperative bacteremia in the ICU: relationship with the level of procalcitonin. Anesteziologiya i reanimatologiya, 4, 22-27.

[11] Nathens, A. B., Rotstein, O. D., Marshall, J. C. (1998). Tertiary Peritonitis: Clinical Features of a Complex Nosocomial Infection. World Journal of Surgery, 22 (2), 158-163. doi: 10.1007/s002689900364

[12] Garner, J. S., Jarvis, W. J., Emori, T. G.; Olmsted, R. (Ed.) (1996). CDC definitions for nosocomial infections. APIC infection control and applied epidemiology: principles and practice. St. Louis: Mosby, 1-20. 
[13] Wittmann, D. H., Schein, M., Condon, R. E. (1996). Management of Secondary Peritonitis. Annals of Surgery, 224 (1), 10-18. doi: 10.1097/00000658-199607000-00003

[14] Dimopoulos, G., Ntziora, F., Rachiotis, G., Armaganidis, A., Falagas, M. E. (2008). Candida Albicans Versus Non-Albicans Intensive Care Unit-Acquired Bloodstream Infections: Differences in Risk Factors and Outcome. Anesthesia \& Analgesia, 106 (2), 523-529. doi: 10.1213/ane.0b013e3181607262

[15] Gostishchev, V. K., Stanoyevich, Yu. S., Aleshkin, V. A., Afanasyev, S. S., Voropayeva, E. A., Shkrob, L. O. et. al. (2007). Tertiary peritonitis: the possibilities of its prevention. Khirurgiya, 9, 15-18.

[16] Ruler, O., Kiewiet, J. J. S., Ketel, R. J., Boermeester, M. A. (2012). Initial microbial spectrum in severe secondary peritonitis and relevance for treatment. European Journal of Clinical Microbiology \& Infectious Diseases, 31 (5), 671-682. doi: 10.1007/s10096-011-1357-0

[17] Bezrodnyi, B. H., Surmasheva, O. V., Iovitsa, A. V., Martynovych, L. D., Moiseienko, A. I. (2011). The choice of empirical therapy in the surgical treatment of patients with destructive forms of appendicitis. Khirurhia Ukrayiny, 1, 17-22.

[18] Rylov, A. I., Kravets, N. S. (2010). Antibiotic therapy of secondary purulent-septic intra-abdominal complications. Materialy XXII zyizdu khirurhiv Ukrayiny, 2, 124-125.

[19] Zubkov, M. N. (2009). Algorithms for antibiotic therapy of severe bacterial infections. Ostryye i neotlozhnyye sostoyaniya $\mathrm{v}$ praktike vracha, 3, 2-7.

[20] Sartelli, M., Abu-Zidan, F. M., Ansaloni, L., Bala, M., Beltran, M. A., Biffl, W. L. et. al. (2015). The role of the open abdomen procedure in managing severe abdominal sepsis: WSES position paper. World Journal of Emergency Surgery, 10 (1), 35. doi: 10.1186/s13017-015-0032-7

[21] Montravers, P., Dupont, H., Gauzit, R., Veber, B., Auboyer, C., Blin, P. et. al. (2006). Candida as a risk factor for mortality in peritonitis. Critical Care Medicine, 34 (3), 646-652. doi: 10.1097/ 01.ccm.0000201889.39443.d2

[22] Nychytaylo, M. E., Medvetskyy, E. B., Bulyk, L. M. (2011). Antibiotic resistance of intraabdominal infections. Klinichna khirurhiya, 4, 65-72.

[23] Montravers, P., Dufour, G., Guglielminotti, J., Desmard, M., Muller, C., Houissa, H. et. al. (2015). Dynamic changes of microbial flora and therapeutic consequences in persistent peritonitis. Critical Care, 19 (1), 70. doi: 10.1186/s13054-015-0789-9

[24] Sandven, P., Qvist, H., Skovlund, E., Giercksky, K. E. (2002). Significance of Candida recovered from intraoperative specimens in patients with intra-abdominal perforations. Critical Care Medicine, 30 (3), 541-547. doi: 10.1097/00003246-200203000-00008

[25] Deykalo, I. M., Makhnits'kyy, A. V., Sokolyk, M. B. (2010). Immunocorrection in the complex treatment of patients with acute abdominal sepsis. Shpytalna khirurhiya, 4, 27-30.

[26] Ballus, J., Lopez-Delgado, J. C., Sabater-Riera, J., Perez-Fernandez, X. L., Betbese, A. J., Roncal, J. A. (2015). Surgical site infection in critically ill patients with secondary and tertiary peritonitis: epidemiology, microbiology and influence in outcomes. BMC Infectious Diseases, 15 (1). doi: 10.1186/s12879$015-1050-5$

[27] Blot, S., Dimopoulos, G., Rello, J., Vogelaers, D. (2008). Is Candida really a threat in the ICU? Current Opinion in Critical Care, 14 (5), 600-604. doi: 10.1097/mcc.0b013e32830f1dff

[28] Chi, H.-W., Yang, Y.-S., Shang, S.-T., Chen, K.-H., Yeh, K.-M., Chang, F.-Y., Lin, J.-C. (2011). Candida albicans versus non-albicans bloodstream infections: The comparison of risk factors and outcome. Journal of Microbiology, Immunology and Infection, 44 (5), 369-375. doi: 10.1016/j.jmii.2010.08.010

[29] Augustin, P., Kermarrec, N., Muller-Serieys, C., Lasocki, S., Chosidow, D., Marmuse, J.-P. et. al. (2010). Risk factors for multidrug resistant bacteria and optimization of empirical antibiotic therapy in postoperative peritonitis. Critical Care, 14 (1), R20. doi: 10.1186/cc8877 